\title{
Humanismo y transversalidad
}

\section{Humanism and transversality}

\author{
Juan Carlos Flores Cornejo \\ Sede Interuniversitaria de Alajuela. \\ tua@hotmail.com
}

\section{Resumen}

Este ensayo trata de analizar algunos elementos necesarios para entender las formas en que el humanismo y la transversalidad convergen mediante la inclusión de sistemas y programas educativos que funcionen desde la última. Se ofrece un panorama del sistema educativo costarricense, evidenciando la posibilidad de llevar a la práctica la transversalidad, para finalmente establecer el humanismo como el tema que podría subsanar las diferencias y lograr la integración de los diferentes saberes. Es así como la mediación pedagógica, desarrollada desde la transversalidad, presente en ejes humanistas, será la conexión entre la adquisición de conceptos, el aprendizaje y la puesta en práctica de los valores sustentados desde el humanismo.

Palabras clave: Transversalidad, humanismo, educación, competencias, valores.

\section{Abstract}

This essay aims to analyze how Humanism and transversality converge on systems and education programs. A panorama of the Costa Rican education system is offered. In this panorama is presented the possibility of carrying out the practice of transversality, to finally establish the Humanism as the topic that could overcome differences and reach the integration of different kinds of knowledge. Thus the pedagogical mediation, developed from transversality, present in humanistic approaches, will be the connection between acquisition of concepts, learning, and putting in practice values based on Humanism. 
Keywords: Transversality, Humanism, education, competences, values.

pesar de ser el humanismo, en su forma básica de acercamiento al concepto,
un paradigma epistémico que entiende la existencia de conocimientos
mayores y menores, así como la designación de lugares donde se produce el conocimiento, considero posible el humanismo como corriente filosófica que pretende el entendimiento del mundo de una forma combinada entre los saberes y no una receta intelectual $o$, en su defecto, una pose académica que justifica las posiciones antagónicas que se tienen con respecto a los otros conocimientos.

Una breve visita al término nos conduce al Renacimiento europeo y nos confirma la posición occidentalista de este, de esta manera que el término explorado por medio del diccionario de la Real Academia, nos remite a tres vertientes básicas de posicionamiento del término: el primero se refiere al "conocimiento de las letras humanas," seguido por el de "movimiento renacentista que propugna el retorno a la cultura grecolatina como medio de restaurar los valores humanos." Estos primeros acercamientos transmiten la idea del conocimiento como forma de estudio, la gnoseología fundamentada como una verdad insondable y conectada al desarrollo del pensamiento occidental. No obstante, la tercera definición es acertada en cuanto no precisa la geopolítica del conocimiento y su locus de enunciación en ningún lugar de occidente, desbordando y ampliando los márgenes del significado al proponernos una "doctrina o actitud vital basada en una concepción integradora de los valores humanos".

Ahora bien, el legado renacentista es ecualizado con una fórmula, si bien simplista, más clara y acorde con las políticas de integración de saberes y conocimientos en la geopolítica de conocimiento actual. Propone, entonces, al humanismo como un saber de saberes y excusa la centralidad del significado por medio de la conjunción disyuntiva del "o"; en dicho caso, será el papel de los sujetos interlocutores el de otorgarle la denotación de equivalencia, alternativa o diferencia.

No obstante, como base desde la cual parte mi argumento de humanismo, propongo la siguiente versión: es la actitud que tenemos hacia los valores humanos lo que nos acerca al humanismo, no la centralidad del saber ni la excusa equívoca de la razón.

El argumento anterior es esencial, si queremos entender la forma como la tecnología y el humanismo deberían converger en la actualidad globalizada, pues es el 
humanismo el que nos permitirá distinguir entre lo mecanizado de la tecnología y dirigirla hacia senderos que nos lleven a la integración del pensamiento filosófico y la razón. La distinción de estas dos vertientes es complicada, pero no imposible, si se dispone del humanismo, de manera primordial, como conducto vertical y horizontal desde el cual se elaboran las teorías científicas para la explicación de la realidad. La razón y la filosofía son un binomio tan complicado como la fe y la razón (fides et ratio) que nos divide y separa, al tiempo que también nos condiciona: el humanismo no debe perder la facilidad de la razón, pero no debe caer en la desesperación de la lógica sin argumentación.

\section{Educación. transversalidad y humanismo}

Los sistemas educativos actuales se enfrentan al reto de ofrecer a la sociedad las herramientas que le permitan resolver las demandas y exigencias de un mundo cada vez más globalizado. No obstante, ante la afluencia desmedida de información, se hace necesario encontrar vías que potencien la relectura del mundo cotidiano a partir de nuestra propia realidad.

La educación ha de encausarse hacia nuevas posibilidades que consientan el desarrollo del individuo en función de la otra persona, como forma de crear espacios de crecimiento democrático que permitan preservar la cultura y los valores nacionales.

Evidentemente, la transversalidad se debería implementar en el sistema educativo costarricense para facilitar el fortalecimiento de los valores democráticos, precisamente por medio de la práctica, conceptualización, entendimiento de los valores, la problematización del conocimiento y la realidad para, consecuentemente, aplicar ese conocimiento a la solución de los problemas. Y anoto que se debería implementar, pues actualmente, a pesar de tener la transversalidad como uno de los elementos del currículo educativo, no se implementa de la manera adecuada en los centros educativos.

El desarrollo histórico de Costa Rica ha permitido que la democracia sea una forma de convivencia social basada en el respeto mutuo, la libertad e igualdad. Asimismo, es una organización jurídico-política que garantiza la participación de la ciudadanía, limita el poder del Estado y su relación con los individuos. 
Esta tradición democrática la recoge la Constitución Política de Costa Rica de 1949 y la enriquece el capítulo V sobre "Derechos y Garantías Sociales", cuyo objetivo es hacer partícipe a la población del bienestar social por medio del "adecuado reparto de la riqueza" (Art. 50). Es elemental comprender que el bien común ha de interpretarse con arreglo al orden institucional como un todo y de conformidad con los valores democráticos (dignidad y libertad del ser humano).

En el ámbito educativo, el carácter social de la Constitución Política de Costa Rica se cristaliza en el capítulo VII titulado "La educación y la cultura". Los artículos contemplados en este apartado son el fundamento legal del sistema educativo nacional. De esta manera, se establece una organización correlacionada desde preescolar hasta la educación superior (Art. 77), disposición que le brinda una integración horizontal y vertical al planeamiento educativo nacional desde una vertiente del pensamiento humanista, que resalta los valores como esenciales en el desarrollo de la nación y la base de la educación estatal.

Aunado a esto, la gratuidad y obligatoriedad de la enseñanza general básica y diversificada se contemplan en el artículo 78, donde se plantea que para hacer realidad la gratuidad de la enseñanza se designe el $6 \%$ del presupuesto nacional para el sector educativo y se prevean mecanismos legales para ayudar a los sectores más necesitados. Este artículo fue retomado en 1973, al establecer la estructura de la educación general básica en tres ciclos de tres años cada uno, a saber: dos ciclos de la educación primaria y uno de enseñanza media.

Con respecto a la libertad de enseñanza (Art. 79), de acuerdo con lo dispuesto por la Sala IV, es un derecho en libertad que el Estado debe estimular, respetar y garantizar. Por otra parte, las libertades se dividen en dos direcciones correlativas; es decir, la libertad de enseñanza encierra el derecho a enseñar (sistema público o privado) y el derecho a aprender. Este último derecho es ejercido por padres y madres y personas tutoras que son quienes eligen el tipo de educación para sus hijos e hijas, moralidad educativa, centros educativos o la formación moral y religiosa (Voto $\mathrm{n} .{ }^{\circ} 3550-92$ ).

Por su parte, el artículo 81 de la Constitución de Costa Rica de 1949 establece que "la dirección de la enseñanza oficial corresponde a un consejo superior integrado como señale la ley, presidido por el Ministerio del ramo" (CONAMAJ, 2000). Es importante recordar que el Estado, como persona jurídica, opera 
mediante órganos que están autorizados constitucionalmente para hacer efectivo el principio de legalidad.

La creación, integración y funcionamiento del Consejo Superior de Educación se logra con la Ley n. ${ }^{\circ} 1362$ de octubre de 1951 (Alfaro, 1999) y la posterior reglamentación de este órgano fue decretada en 1953 (Decreto Ejecutivo n. $^{\circ}$ 14) (Alfaro, 1999). Ambas disposiciones hacen realidad la norma constitucional, al tiempo que apoyan los valores fundamentales de la democracia nacional.

\section{Ley fundamental de educación}

La Ley Fundamental de Educación de 1957 estipula los fines de la educación costarricense y dota de una estructura al sistema educativo nacional, además de que le imprime un carácter democrático cuando establece que "todo habitante de la Republica tiene derecho a la educación" (Alfaro, 1999, p. 9). Es obvio que se hace referencia a la igualdad de oportunidades para ejercer, en libertad, el derecho a aprender como el derecho a la enseñanza.

La libertad es un derecho que consagra la Constitución del país (1949, en el articulo 20, y un pilar fundamental de la democracia, puesto que implica el derecho de cada uno de participar en la vida política según sus intereses y capacidades; el reconocimiento del derecho de cada uno de participar en la vida política según sus intereses y capacidades (Capella, 2003b). El individuo, al ejercer la libertad, adquiere un sentido de responsabilidad individual y social. Por otra parte, el respeto a la dignidad humana es el objetivo común de las sociedades democráticas dentro de las cuales las instituciones políticas aseguran el desarrollo de los valores personales y sociales. En nuestro caso, el desarrollo individual y social de la personalidad se contempla en el artículo 2, incisos a y b de la Ley Fundamental de Educación (1957).

Dentro de los fines de la educación costarricense, está implícito el concepto de formación humana o humanización de la persona. Flores Ochoa (2000) expone "formar, pues, a un individuo en su estructura más general es facilitarle que asuma en su vida su propia dirección racional, reconociendo fraternalmente a sus semejantes el mismo derecho y la misma dignidad" (p. 111).

El concepto esbozado anteriormente reúne tres elementos substanciales para la educación de los seres humanos, a saber: la condición de perfectibilidad de la 
persona humana, la razón como finalidad de la educación y el cómo lograremos la comprensión del proceso de enseñanza aprendizaje. Al respecto, el artículo 3 de la Ley Fundamental de Educación, al tomar en cuenta la parte biológica (salud física), psicológica (salud mental, aptitudes y diferencias), intelectual (conocimientos, técnicas y capacidad productora) y moral (valores estéticos, éticos, religiosos y cívicos) del individuo, vislumbra el desarrollo individual del ser costarricense de forma más que integral. De la misma manera, la educación pública se plantea como "un proceso integral correlacionado en sus diversos ciclos" (Art. 4, LFE, 1957). La correlación, de esta manera, permite asegurar la continuidad y unidad del proceso de enseñanza aprendizaje de acuerdo con las necesidades psicobiológicas del estudiantado.

El proceso de formación individual se revierte socialmente cuando la educación permite preservar la cultura nacional y universal. Se expone que se debe conservar y ampliar la herencia cultural, cuando se comparte el conocimiento sobre la historia del hombre, las grandes obras de la literatura y los conceptos filosóficos fundamentales, la vida familiar y los valores de nuestra sociedad (LFE, 1957).

Como ha quedado claro, los sistemas educativos democráticos promueven el desarrollo y la conservación de la identidad nacional; obviamente, un proyecto de nación requiere la elección de un proyecto histórico concreto. En el caso de Costa Rica, la década de las cuarentas fue de reformas sociales y de conflictos políticos que culminaron con la lucha armada de 1948. A partir de este hecho, se logra consolidar un estado de derecho (jurídico-político) y se elevan a rango constitucional las reformas sociales. La segunda mitad del siglo XX estuvo determinada por los ideales socialdemócratas, por lo cual el sistema educativo nacional hunde sus raíces en el humanismo socialcristiano.

Evidentemente, el proyecto político responde a las necesidades históricas de las que surge, de ahí que exista coherencia entre el sistema político y la educación. De hecho, al analizar la LFE de 1957, se puede apreciar un claro interés por la educación democrática como rasgo distintivo de la identidad nacional, la democracia como garantía del derecho individual y social del pueblo costarricense. En este proyecto, fue imprescindible la educación en cuyas actividades privara "un ambiente democrático, de respeto mutuo y de responsabilidad" (Art. 10). Igualmente se estableció que la educación media debía "preparar para la vida cívica y el ejercicio responsable de la libertad, procurando el conocimiento 
básico de las instituciones patrias y de las realidades económicas y sociales de la Nación" (Art. 14, inciso d).

Aunado al proyecto histórico, se inserta la responsabilidad del individuo de comprender su realidad social, económica y política, por lo cual, los programas han de adecuarse a las condiciones y necesidades del país (LFE, Art. 9), para fortalecer la participación ciudadana en un clima de respeto y justicia social. Un sistema educativo con estas características permite la efectividad del hecho educativo (formación) a través de los procesos de personalización y socialización del individuo. Entenderemos, pues, el proceso de individuación de los seres humanos por medio de la integración (cohesión en su funcionamiento), autodeterminación (responsabilidad y compromiso) y el autocontrol como características de maduración personal. En tanto, la socialización es la interacción de la persona con la sociedad, lo que le permite asimilar las pautas, las normas y las costumbres de esa sociedad (Fermoso, 2003).

A lo largo de este ensayo hemos hecho referencia al sentido de la educación nacional y visto la congruencia entre el proyecto histórico político y social del Estado costarricense, instituido por medio del aparato jurídico y las aspiraciones del sistema educativo nacional. Esta realidad es más perceptible cuando leemos a Flores Ochoa (2000):

La temporalidad es la determinación esencial de la conciencia, su historicidad, la historicidad del ser ahí, pues ya no se trata de una conciencia absoluta, desligada y naturalmente libre sino constituida por su factibilidad esencial... Es solo desde su situación... desde su pasado y desde la tradición como podrá proyectarse realizándose bajo la forma de "comprender", como la forma originaria de ser-ahí. Comprender es conocer con solvencia las propias posibilidades del poder ser, comprender es siempre comprenderse a sí mismo. Se comprende lo otro no porque existe homogeneidad entre el cognoscente y lo conocido, sino porque ambos participan de la historicidad como su peculiaridad esencial, como su modo de ser. El que hagamos historia es solo porque nosotros mismos somos "históricos", porque la historicidad del hombre en su movilidad del esperar y el olvidar es la condición de que podamos de alguna manera actualizar el pasado, no desde motivaciones e intereses subjetivos, sino por nuestra real inserción en las tradiciones que determinan nuestro presente, nuestra finitud histórica original... 
Las metas de la pedagogía son también productos históricos de la reflexión humana condicionada en cada época por la tradición, los prejuicios, los proyectos y las posibilidades futuras en que está inscrito el investigador pedagógico y los maestros en general (pp. 190-191).

\section{Política Educativa hacia el Siglo XXI}

Para garantizar la no injerencia política partidista en la gestión educativa y atendiendo la disposición constitucional del artículo 81, se creó el Consejo Superior de Educación en 1951, concebido como un órgano técnico que orienta y dirige la educación pública, el cual, dentro de la estructura administrativa del MEP, se encuentra en el área determinativa.

Hablamos de área determinativa, porque de acuerdo con el artículo 4 de la Ley n. ${ }^{\circ}$ 1362 (1951), al Consejo Superior de Educación le corresponde conocer:

a. Los planes para incrementar la Educación Pública.

b. Los proyectos de ley, reglamentos, planes de estudio y programas a que deben someterse los establecimientos educativos y resolver sobre los problemas de correlación e integración del sistema educativo.

c. Los planes para la preparación, el perfeccionamiento y el estímulo del personal docente... (Alfaro, 1999, p. 42).

La reglamentación del Consejo Superior de Educación se realiza a través del Decreto Ejecutivo n. ${ }^{\circ} 14$ de 1953 (reformado por el Decreto n. ${ }^{\circ} 3$ de 1967, n. ${ }^{\circ} 6$ de 1968 y n. ${ }^{\circ} 19635$ de 1990). En el capítulo II sobre Funciones y Atribuciones del Consejo Superior de Educación, se instituye lo siguiente:

Artículo, 4. Para establecer la dirección general de la enseñanza y la cultura, el Consejo tendrá las siguientes atribuciones, de acuerdo con el artículo 4 de la Ley: en los incisos siguientes:

a. Incrementar la Educación Pública, para lo cual podrá contar con el asesoramiento que estime necesario de los técnicos y de los funcionarios.

b. Procurar la integración del sistema educativo del país-legislación, planes de estudio, programas, administración, etc. Para tal efecto podrá pedir a los funcionarios del ramo que formulen los proyectos y, si lo cree necesario, los llamará para oír informaciones. 
c. Formular los planes para la formación docente en los institutos del Estado, y para el perfeccionamiento y el estímulo del Personal Docente. (Alfaro 1999, p. 45).

Aquí se citan los artículos e incisos de la Ley y el Decreto que interesan en este trabajo. La información anterior permite observar el carácter vertical del derecho educativo, a saber: orden constitucional (leyes), las disposiciones ejecutivas (decretos) que regulan la relación del Estado y servidoras y servidores públicos y los reglamentos propios de cada ministerio. Simultáneamente, podemos establecer niveles de horizontalidad en ese conjunto de normas, pero especialmente entre la ley de 1951 y la de 1953. Lo realmente importante es que las decisiones o determinaciones en política educativa son facultad propia del Consejo Superior de Educación, presidido por el ministro o ministra.

En la década de los noventas, los cambios en la orientación política del Estado obligan al sistema educativo nacional a buscar soluciones a los problemas administrativos, curriculares, infraestructurales, financiamiento, entre otros; la posición adoptada fue la del uso racional de los recursos humanos y materiales. Las acciones del Ministerio de Educación se orientaron hacia el desarrollo de una política curricular y la elaboración de programas de estudio.

La Política Educativa hacia el Siglo XXI del Ministerio de Educación Pública nace de la urgente necesidad de releer la realidad nacional en un contexto mundial de cambios acelerados, novedosos y diversos, donde el plano educativo brinde la posibilidad de apropiarse del saber "aprender a aprender". El proceso de enseñanza - aprendizaje ha de centrarse en el alumnado para posibilitar un aprendizaje permanente ante la inusitada afluencia de datos que tenemos hoy con la democratización del conocimiento mediante las tecnologías de información y comunicación.

La obligación del personal docente es la del compromiso permanente, mediante la formación y actualización constante del conocimiento, además de una revisión continua del quehacer cotidiano. Se plasma en esta actitud el carácter humanista de la educación, en la medida en que implica un proceso de perfeccionamiento de la naturaleza del ser humano y, por lo tanto, la personalización del individuo ante el mundo a través del pensamiento crítico y creativo, la libertad de acción de la persona en un ambiente de respeto y solidaridad con el colectivo y, por último, la capacidad propositiva del ser humano ante una situación particular.

Además del humanismo, el racionalismo es parte de los principios de la política educativa vigente, puesto que, al concebir al individuo como un ser eminentemente 
racional, este es capaz de discernir, de tomar decisiones en función de sus intereses individuales y sociales. Es precisamente la razón la que ha permitido al ser humano transformar el medio natural y social en estructuras más complejas. En este sentido, la capacidad de conocer parte de la persona humana y el ser humano es el fin último del conocimiento.

El constructivismo aúna el pensamiento filosófico humanista y racionalista, puesto que en el centro del proceso de enseñanza - aprendizaje se encuentra el alumnado como objeto y sujeto de la educación. El proceso continuo y progresivo del aprendizaje facilita el aprender significativamente, pues toma el conocimiento previo y lo redimensiona con los conocimientos nuevos. Estos estarán mediatizados por las motivaciones e intereses del estudiantado, al mismo tiempo que se vinculan con el contexto real de la comunidad educativa.

Los lineamientos de la Política Educativa hacia el Siglo XXI pretenden desarrollar la formación de personas dignas, libres, justas y espirituales, mientras se identifiquen con los valores democráticos de la sociedad. Dentro de este marco de acción, el compromiso de docente es el de identificar, seleccionar y organizar los objetivos para integrarlos correlacionadamente en las diferentes materias académicas, en un ambiente de respeto, colaboración y democracia.

Es indudable que existe una preocupación por hacer efectivos los fines del sistema educativo nacional a través de los lineamientos de la política educativa vigente: por lo tanto, tiene un carácter legal ineludible.

\section{Los temas transversales}

Entre el 2000 y el 2002, el Ministerio de Educación Pública analiza y define el marco conceptual de la transversalidad como herramienta pedagógica (MEP, 2004) que posibilita el acceso a la información, así como el desarrollo de valores, actitudes, habilidades y destrezas del estudiantado con el objeto de que tenga la capacidad de integrarse a las exigencias de un mundo globalizado.

La formación de valores es el eje de la transversalidad, ya que constituyen aspectos importantes de la formación de estudiantes y, por ende, son parte del planeamiento didáctico. Debido a que cada sociedad tiene características muy particulares y diversas, los valores se abordan por medio de los temas transversales que reflejan las necesidades de la sociedad. De acuerdo con este criterio, nuestro sistema 
educativo define los siguientes temas transversales: a) cultura ambiental para el desarrollo sostenible; b) educación para la salud; c) educación integral para la sexualidad; y c) vivencia de los derechos humanos para la democracia y la paz.

La transversalidad parte de que los valores desarrollan en los seres humanos competencias, es decir, "un conjunto integrado de conocimientos, procedimientos, actitudes y valores, que permiten un desempeño satisfactorio y autónomo ante situaciones concretas de la vida personal y social" (MEP, 2004, p. 5), cuyo carácter interdependiente permite profundizar las dimensiones afectivas (procesos de individualización, personalización) y cognitivas (procesos de conocimiento), en procesos reflexivos a nivel ético de la realidad y la convivencia social que les permite responsabilizarse socialmente.

De acuerdo con lo anterior, cada uno de los temas transversales propuestos implementa competencias y contenidos específicos que, al estar interrelacionados, permiten el desarrollo integral del ser humano. La integralidad e interdependencia de las competencias permite hablar de competencias transversales, puesto que empapan el currículo de forma horizontal y vertical, al punto que podemos hablar de transdisciplinaridad e interdisciplinaridad; su puesta en práctica depende del soporte de otras disciplinas (MEP, 2004).

Cada tema transversal cuenta con conceptos, habilidades, valores y actitudes. Cuando hablamos de conceptos nos referimos a contenidos, ideas, principios, significaciones, hechos, referencias y relaciones; estos se gradúan de acuerdo con el desarrollo cognitivo del estudiantado y al nivel escolar en que se encuentra (Magendzo, 2002).

Las habilidades son las destrezas y capacidades que tienen los alumnos y alumnas para aplicar el conocimiento a la resolución de problemas del tema transversal, o bien, a un problema cotidiano. La resolución de problemas cuenta con una actitud del ser humano producto de la valoración ética o concepción de mundo de las personas y de la sociedad a la que pertenecen; en nuestro caso, las actitudes estarían enmarcadas por los valores democráticos.

La efectividad de la aplicación de los temas transversales está en relación directa con la apropiación de la propuesta teórica por parte del currículo, la organización educativa, el personal docente y, por supuesto, la experiencia práctica. 
Las propuestas educativas han estado permeadas por la necesidad de preparar a los individuos para su incorporación al aparato productivo; en la actualidad, las tendencias educativas plantean la formación del ser humano en dos planos: el interno, que busca preparar para el ejercicio de la ciudadanía, y el externo, que trata de educar con el fin de estar en la capacidad de competir en un mundo globalizado (Magendzo, 2002). La formación de personas integrales, cuyos valores y competencias (destrezas, habilidades) les permitan vivir en una sociedad globalizada, revela el carácter holístico, axiológico, interdisciplinario y penetrante de la transversalidad.

Es holístico puesto que parte del interés de respetar el desarrollo psicológico (estructura cognitiva y afectiva) del estudiantado (Torres, 1996). En un primer momento el concepto de globalización, aplicado a la educación, surge como metodología de enseñanza justificada por investigaciones psicológicas que confirman que la percepción de los niños y las niñas es global (percepción sincrética: E. Claparéde, Ernest Renan, Max Wertheimer, Wolfgang Kohler, Kurt Koffka y Felix Krueger). Posteriormente, la Gestalt se dedicó al

El estudio de la percepción y constatan claramente que las percepciones humanas se presentan con unidad, como un todo $\mathrm{y}$, por consiguiente, con significado (...) Toda actividad perceptiva está condicionada por las experiencias anteriores de quien percibe; las experiencias anteriores son la base de las siguientes percepciones, de la construcción de totalidades significativas (Torres, 1996, p. 37).

De esas investigaciones se desprende la importancia de la comprensión en los procesos de enseñanza y aprendizaje con una clara diferenciación entre el conocimiento reproductivo y el conocimiento productivo, es decir, la organización conceptual (perceptiva) de un problema y la resolución de este.

Otras corrientes educativas que trabajan la totalidad de los aprendizajes son las teorías cognitivas (Henri Wallop, Georges-Henri Luquet). Nuevos avances se dieron con Ovide Decroly que desarrolla los centros de interés o ideas-eje como punto de unión de distintos intereses psicológicos y sociales, entre otros (Torres, 1996).

El personal docente en el aula ha de procurar la implementación de los temas transversales en su planeamiento didáctico, propiciar una actitud crítica de la realidad incorporándolo en las actividades de mediación y evaluación. Es un 
proceso que se justifica porque el aprendizaje es eminentemente social. Al respecto, Torres (1998) anota:

Como un proceso profundamente social, necesita adaptar las estrategias y contenidos de los proyectos curriculares al contexto histórico y cultural específico en el que vive en alumnado; esta es la manera de Ilegar a utilizar sus conceptos espontáneos, aquellos que son fruto de las interacciones cotidianas en un medio social, con los nuevos conceptos que las instituciones docentes les facilitan. (p. 43)

Lo anterior nos conduce al carácter interdisciplinario de la transversalidad. Desde el conocimiento científico existen tres tendencias:

- La primera es la especialización.

- La segunda son disciplinas que comparten objetos de estudio o metodologías.

- Por último, los equipos de trabajo interdisciplinario.

Actualmente, la (última opción cobra mayor interés, debido fundamentalmente a que los niveles de complejidad de los fenómenos sociopolíticos y económicos exigen un examen desde distintas ópticas).

Si el contexto es importante para el aprendizaje significativo (percepción global), también lo es el conocimiento como totalidad comprensiva, en tanto que uno de los elementos fundamentales de la educación es la culturalización del ser humano (socialización), sin dejar de lado la personalización del individuo. Torres (1996) plantea que la interdisciplinaridad:

Implica una voluntad y un compromiso de elaborar un marco más general en el que cada una de las disciplinas en contacto son a la vez modificadas y pasan a depender claramente una de otras. Aquí se establece una interacción entre dos o más disciplinas, lo que dará como resultado una intercomunicación y un enriquecimiento recíproco y, en consecuencia, una transformación de sus metodologías de investigación, una modificación de conceptos, de terminologías fundamentales, etc. Entre las distintas materias se dan intercambios mutuos y recíprocas integraciones; existe un equilibrio de fuerzas en las relaciones que se establecen. (p. 75)

Es necesario tener clara la noción de interdisciplinaridad, pues la transversalidad, en nuestro sistema educativo, está articulada en torno a los cuatro temas 
transversales (ambiente, sexualidad, salud y derechos humanos), a partir de los cuales es posible establecer correlaciones entre las asignaturas, no solo en lo conceptual, sino también en el plano metodológico. Este es un trabajo que está por realizarse y que debe procurar aunar conceptos y procedimientos para fortalecer los procesos de enseñanza - aprendizaje.

Lo más interesante es que la transversalidad supera la interdisciplinaridad, al volverse transdisciplinaria en la medida en que rebasa el ámbito de las disciplinas (asignaturas) y las correlaciones entre esas disciplinas: se vuelve totalidad en sí. Torres (1996) habla de omnicomprensión, porque Ia percepción de los objetivos, en nuestro caso, la transversalidad, constituye un objetivo y una finalidad educativa.

De lo anterior, caemos irremediablemente en otra de las características de la transversalidad: la axiología. Para hablar de los valores debemos recurrir a la fundamentación antropológica y sociológica de la educación; educar es formar y en ese proceso de formación de los individuos se afirman y realiza dentro de los valores de una sociedad concreta (fines educativos en función de las necesidades sociales y el modelo de ser humano que la sociedad sueña) (Fermoso, 2003).

Queda claro que los valores determinan los fines educativos. Estos, a su vez, definen los objetivos educativos que se concretan en actitudes que son las habilidades manifiestas en las consideraciones de los individuos, por medio de sus actos con respecto a los valores sociales (Fermoso, 2003).

Pero las actitudes de los seres humanos son producto del conocimiento que adquieren conscientemente: la conciencia permite comprender la realidad vivida, es decir, el discernimiento humano es producto de una cultura particular (visión de mundo) y cada ser humano se apropia de ese acervo autoconstruyendo permanentemente su conciencia (Florez, 2000). La conciencia, obviamente, está determinada por los valores. Es importante hacer notar que la transversalidad posibilita la concreción de los fines educativos de nuestro sistema educativo nacional.

A la postre, los temas transversales facilitan la puesta en práctica del cuarto postulado que enunció la Organización de las Naciones Unidas para la Educación, la Ciencia y la Cultura (UNESCO): aprender a convivir dentro de un clima de cooperación y de solidaridad que presupone el respeto por las diferencias (económicas, políticas, religiosas, sociales, entre otras) (Ander-Egg, 2001). Esto solo es factible por medio del establecimiento de los marcos de referencia con la comunidad nacional, dentro 
de planes de estudio que, concienzudamente, incluyan y pongan en práctica el humanismo como eje transversal dentro de sus currículos.

\section{Referencias}

Alfaro, D, (Comp.). (1999). Código de normas y reglamentos sobre educación (2a. ed.). San José, Costa Rica: Editorial Porvenir.

Ander-Egg, E. (2001). Los desafíos de la educación en el siglo XXI. RosarioSanta Fe, Argentina: Ediciones Homo Sapiens.

Capella, R. J. (2003b). Enfoque antropológico de la educación. Planteamiento para la formulación de una teoría. Teoría de la educación. San José, Costa Rica: Editorial UNED.

Comisión Nacional para el Mejoramiento de la Administración de Justicia (CONAMAJ). (2001). Constitución de la Republica de Costa Rica: Texto oficial. San José, Costa Rica: Imprenta Nacional.

Fermoso, P. (2003). Introducción epistemológica: Concepto de teoría y filosofía de la educación. Teoría de la educación. San José, Costa Rica: Editorial UNED.

Florez, R. (2000). Hacia una pedagogía del conocimiento (2a ed.). Bogotá, Colombia: Mc. Graw Hill Interamericana.

Magendzo, A. (2002). Los temas transversales en el trabajo de aula. Ministerio de Educación Pública. División de Desarrollo Curricular, Fondo de Población de las Naciones Unidas. San José, Costa Rica.

Ministerio de Educación Pública (MEP) (1994). Política Educativa Hacia el Siglo XXI. San José, Costa Rica: Autor.

Oradisón, M. (2000). La transversalidad en la educación moral: sus implicancias y alcances. Organización de Estados Iberoamericanos para la Educación, la Ciencia y la Cultura.

Sarramona, J. (1997). Fundamentos de educación. Barcelona, España: Editorial CEAC. 
Torres, J. (1996). Globalización e interdisciplinaridad: el currículo integrado (2a ed.). Madrid, España: Ediciones Morata.

Torres, J. (1998). El currículum oculto (6a ed.). Madrid, España: Ediciones Morata.

Tuvilla R. J. (2000). Reformas educativas, transversalidad y derechos humanos. Educación Internacional para la Paz. Recuperado de http:// www.eip.org 